\title{
Article \\ A Limited-View CT Reconstruction Framework Based on Hybrid Domains and Spatial Correlation
}

\author{
Ken Deng (D), Chang Sun (D), Wuxuan Gong ${ }^{(D)}$, Yitong Liu *(D) and Hongwen Yang (D)
}

check for

updates

Citation: Deng, K.; Sun, C.; Gong, W.; Liu, Y.; Yang, H. A Limited-View CT Reconstruction Framework Based on Hybrid Domains and Spatial Correlation. Sensors 2022, 22, 1446. https://doi.org/10.3390/s22041446

Academic Editors: M. Jamal Deen, Subhas Mukhopadhyay, Yangquan Chen, Simone Morais, Nunzio Cennamo and Junseop Lee

Received: 7 December 2021 Accepted: 9 February 2022

Published: 13 February 2022

Publisher's Note: MDPI stays neutral with regard to jurisdictional claims in published maps and institutional affiliations.

Copyright: (c) 2022 by the authors. Licensee MDPI, Basel, Switzerland. This article is an open access article distributed under the terms and conditions of the Creative Commons Attribution (CC BY) license (https:// creativecommons.org/licenses/by/ $4.0 /)$.
Institute of Wireless Theories and Technologies Laboratory, Beijing University of Posts and Telecommunications, Haidian, Beijing 100876, China; arieldeng@bupt.edu.cn (K.D.); sc1998@bupt.edu.cn (C.S.); gongwuxuan@bupt.edu.cn (W.G.); yanghong@bupt.edu.cn (H.Y.)

* Correspondence: liuyitong@bupt.edu.cn

\begin{abstract}
Limited-view Computed Tomography (CT) can be used to efficaciously reduce radiation dose in clinical diagnosis, it is also adopted when encountering inevitable mechanical and physical limitation in industrial inspection. Nevertheless, limited-view CT leads to severe artifacts in its imaging, which turns out to be a major issue in the low dose protocol. Thus, how to exploit the limited prior information to obtain high-quality CT images becomes a crucial issue. We notice that almost all existing methods solely focus on a single CT image while neglecting the solid fact that, the scanned objects are always highly spatially correlated. Consequently, there lies bountiful spatial information between these acquired consecutive CT images, which is still largely left to be exploited. In this paper, we propose a novel hybrid-domain structure composed of fully convolutional networks that groundbreakingly explores the three-dimensional neighborhood and works in a "coarse-to-fine" manner. We first conduct data completion in the Radon domain, and transform the obtained fullview Radon data into images through FBP. Subsequently, we employ the spatial correlation between continuous $\mathrm{CT}$ images to productively restore them and then refine the image texture to finally receive the ideal high-quality CT images, achieving PSNR of 40.209 and SSIM of 0.943. Besides, unlike other current limited-view CT reconstruction methods, we adopt FBP (and implement it on GPUs) instead of SART-TV to significantly accelerate the overall procedure and realize it in an end-to-end manner.
\end{abstract}

Keywords: CT image reconstruction; low dose protocol; adversarial autoencoder; deep learning; hybrid domain; spatial correlation; inverse problems

\section{Introduction}

Computed Tomography (CT) [1] is diffusely known as an approach to exhibit precise details inside the scanned object [2], thus is applied to a wide range of applications including clinical diagnosis, industrial inspection, material science and biomedicine [3,4]. In addition, the raging epidemic caused by the Corona Virus Disease 2019 (COVID-19) has made CT known to the public as an efficacious auxiliary technology. Nevertheless, the associated $\mathrm{x}$-ray radiation dose brings potential risk of cancers [5], which has drawn wide attention. Consequently, the demand of radiation dose reduction is becoming more and more acute under the principle of ALARA (as low as reasonably achievable) [6-10].

Generally, Low-dose Computed Tomography (LDCT) can be realized through two strategies including current (or voltage) reduction [11,12] and projection reduction [13-15]. The first strategy aims to lower the x-ray exposure in each view, while it greatly suffers from the increased noise in projections. Although the second strategy can avoid the above problem and realize the additional benefit of accelerated scanning and calculation, it gives rise to severe image quality deterioration of increased artifacts due to its lack of projections. In this paper, we will focus on obtaining high-quality CT images from limited-view CT with inadequate scanning angle.

Researchers have proposed various CT image reconstruction algorithms in the past few decades, but when it comes to LDCT reconstruction, the problem becomes challenging. 
Traditional analytical reconstruction algorithms, such as FBP [16], have high requirements for data integrity. When the radiation dose is reduced, artifacts in reconstructed images will increase rapidly [17]. Compared with analytical reconstruction algorithms, iterative reconstruction algorithms can obtain better performance, while suffering from higher complexity. Model-based iterative reconstruction (MBIR) algorithm [18], combines the modeling of some key parameters to perform high-quality reconstruction of LDCT. Using image priors in MBIR can effectively improve the image reconstruction quality of LDCT scans [14,19], while still have the high computational complexity.

In addition, diverse regularization methods have played a crucial role in CT reconstruction, which is a typical inverse problem. The most prevailing regularization method is the total variation (TV) method [20]. In the light of TV, researchers came up with more reconstruction methods, such as TV-POCS [21], TGV [22] and SART-TV [13] which was proposed on the basis of SART [23]. Those algorithms can suppress image artifacts to a certain extent so as to improve imaging quality. In addition, dictionary learning is often used as a regularizer in MBIR algorithms [24-27], and multiple dictionaries are beneficial to reducing artifacts caused by limited-view $\mathrm{CT}$ reconstruction.

With the development of computing power, deep learning-based methods [28-34] have been applied to the restoration of LDCT reconstructed images in recent years. The methods can be roughly divided into the below three categories.

Image inpainting algorithms usually reconstruct the damaged Radon data into the damaged image with artifacts through regular methods, such as FBP, then reduce the artifacts and noises in the image domain. Lots of researchers are currently using convolutional neural network (CNN) and deep learning architecture to perform this procedure [4,35-44]. Zhang et al. [35] proposed a data-driven learning method based on deep CNN. REDCNN [4] combines the autoencoder, deconvolutional network and shortcut connections into the residual encoder-decoder CNN for LDCT imaging. Kang et al. [36] applied deep CNN to the wavelet transform coefficients of LDCT images, used directional wavelet transform to extract the directional component of artifacts. Wang et al. [39] developed a limited-angle translational CT (TCT) image reconstruction algorithm based on U-Net [40]. Since Goodfellow et al. proposed Generative Adversarial Nets (GAN) [42] in 2014, GAN has been widely used in various image processing tasks, including the post-processing of CT images. Xie et al. [43] proposed an end-to-end conditional GAN with joint loss function, which can effectively remove artifacts.

Sinogram inpainting algorithms firstly restore the missing part in the Radon domain, then reconstruct it into the image domain to get the final result [45-49]. Li et al. [45] proposed an effective GAN-based repairing method named patch-GAN, which trains the network to learn the data distribution of the sinogram to restore the missing sinogram data. In another paper [46], Li et al. proposed SI-GAN on the basis of [37], using a joint loss function combining the Radon domain and the image domain to repair "ultralimited-angle" sinogram. In 2019, Dai et al. [47] proposed a limited-view cone-beam $\mathrm{CT}$ reconstruction algorithm. It slices the cone-beam projection data into the sequence of two-dimensional images, uses an autoencoder network to estimate the missing part, then stack them in order and finally use FDK [50] for three-dimensional reconstruction. Anirudh et al. [48] transformed the missing sinogram into a latent space through a fully convolutional one-dimensional CNN, then used GAN to complement the missing part. Dai et al. [49] calculated the geometric image moment based on the projection-geometric moment transformation of the known Radon data, then estimated the projection-geometric moment transformation of the unknown Radon data based on the geometric image moment.

Sinogram inpainting and image refining algorithms firstly restore the missing part in the Radon domain, then reconstruct the full-view Radon data into the image domain so as to finely repair the image to obtain higher quality [51-55]. In 2017, Hammernik et al. [51] proposed a two-stage deep learning architecture, they first learn the compensation weights that account for the missing data in the projection domain, then they formulate the image restoration problem as a variational network to eliminate coherent streaking artifacts. 
Zhao et al. [52] proposed a GAN-based sinogram inpainting network, which achieved unsupervised training in a sinogram-image-sinogram closed loop. Zhao et al. [53] also proposed a two-stage method, firstly they use an interpolating convolutional network to obtain the full-view projection data, then use GAN to output high-quality CT images. In 2019, Lee et al. [54] proposed a deep learning model based on fully convolutional network and wavelet transform. In the latest research, Zhang et al. [55] proposed an end-to-end hybrid domain $\mathrm{CNN}$ (hdNet), which consists of a CNN operating in the sinogram domain, a domain transformation operation, and a $\mathrm{CNN}$ operating in the image domain.

However, we cannot help but notice that, when it comes to image restoration, all the methods above merely focus on a single CT image while neglecting the solid fact that the scanned object are often spatially continuous. On account of that, these obtained consecutive CT images are always highly correlative, which leads to copious spatial information hidden between them that is still largely left to be explored. Consequently, we propose a novel two-step cascaded model in the second stage which concentrates on groundbreakingly utilizing the strong spatial correlation between consecutive CT images. So as to break the limit of feature extraction in the two-dimensional space and dig deep into the three-dimensional spatial neighborhood.

These two domains are also combined in our method to amalgamate their respective strengths for high-quality CT reconstruction results, which leads to our proposed threestage structure. Specifically, we firstly conduct data completion in the Radon domain to acquire the full-view CT data, and then reconstruct it into images through FBP. Subsequently, image restoration and artifacts removal are accomplished in a "coarse-to-fine" [56] manner with the combination of stage two and stage three.

It is also worth mentioning that, unlike other current prevailing limited-view CT reconstruction methods [39], we adopt FBP [16] (and implement it on GPUs) instead of SART-TV [13] to speed up the overall procedure. Besides, since our method actually consists of fully convolutional networks, it does not limit the resolution of input images, thus can be well generalized to various datasets. In our experiments, we compare our algorithm with other methods under four sorts of limited-view CT data, exhibiting its prominent performance and robustness.

The organization of this paper is as follows, Section 2 presents our proposed method in detail, Section 3 exhibits the experimental results and corresponding discussion, and conclusion is stated in Section 4.

\section{Methods}

In this work, we propose a hybrid-domain limited-view CT reconstruction method, and its overall three-stage structure is shown in Figure 1. In the first stage, after the limited-view Radon data is preprocessed, we fed it into the Adversarial Autoencoder (AAE) established for data restoration, so as to acquire high-quality full-view Radon data, which is then transformed into images through FBP. In stage two, these CT images are concatenated into groups and then sent into our proposed Spatial Adversarial Autoencoder (Spatial-AAE) to perform image inpainting based on strong spatial correlation between consecutive CT images, which can manage to eliminate almost all the artifacts from the original limited-view CT images. However, we notice that the image texture of these restored CT images is still not precise enough compared to the ground truth CT images. Therefore, utilizing the idea of "coarse-to-fine" [56-59] in deep learning, we establish the Refine Adversarial Autoencoder (Refine-AAE) in the third stage to refine the image texture in patches, and eventually obtain the ideal high-quality CT images which are not only artifact-free, but also have fine image texture. 


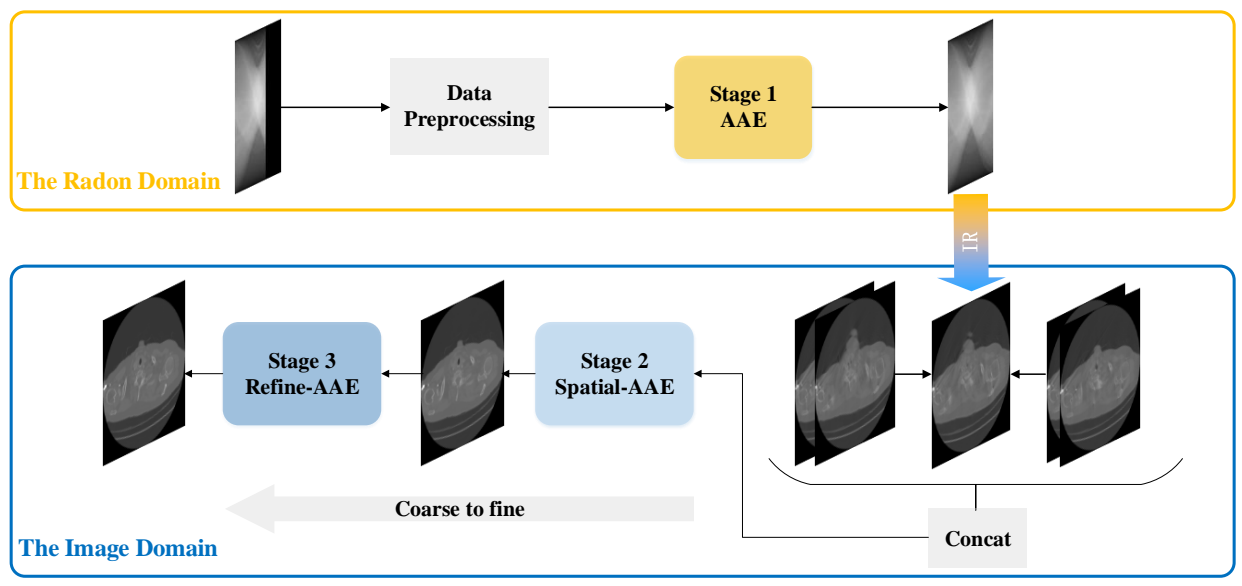

Figure 1. The overall architecture of our proposed method.

\subsection{Preliminaries and Discussion}

\subsubsection{How to Maximize the Limited Prior Information through Data Preprocessing}

In order to obtain more valuable data from the limited prior information, we refer to [38] and adopt the data preprocessing method shown in Figure 2. For the limited-view Radon data $\mathbf{R}_{l v}$, we first convert it into the image $\mathbf{I}_{\text {recon }}$ through inverse radon transformation, and then adopt Radon transformation to transform $\mathbf{I}_{\text {recon }}$ into the full-view Radon data $\mathbf{R}_{f v}$. Subsequently, $\mathbf{R}_{f v}$ is cropped for preliminary completion of the missing part in $\mathbf{R}_{l v}$, so as to obtain the merged full-view Radon data $\mathbf{R}_{\text {merge }}$. In this way, we manage to efficaciously utilize the existing data for better restoration results, which is proved in our experimental results from Section 3.

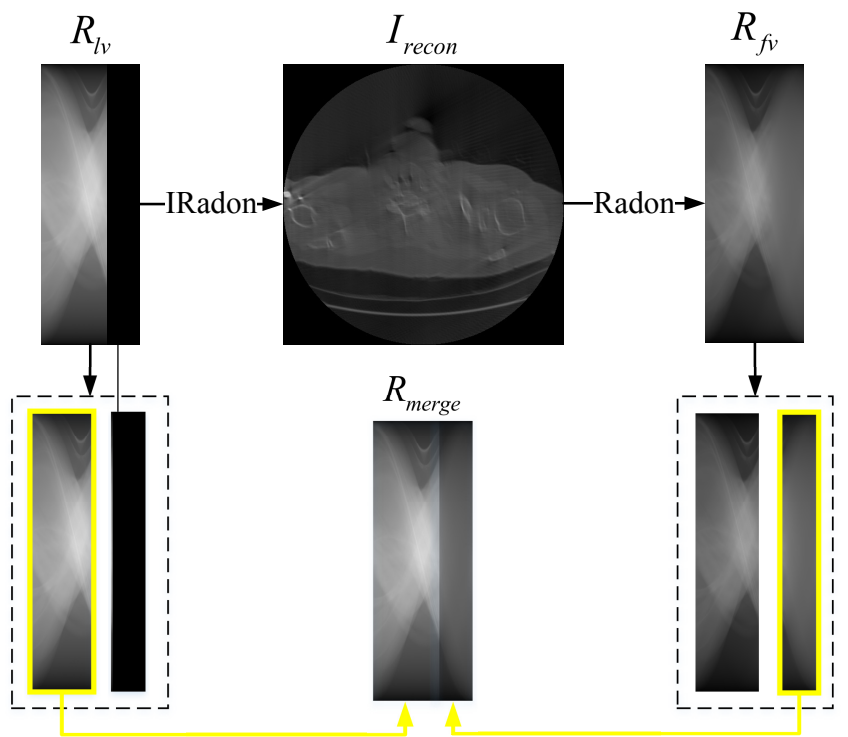

Figure 2. Workflow of data preprocessing.

\subsubsection{How Does Spatial Correlation Help Remove Artifacts}

As we mentioned above, since the scanned objects are always spatially continuous, the consecutive CT images obtained from them also have strong spatial coherence. Consequently, these continuous CT images can be regarded as successive frames from a video clip which have been proved to contain much more information than a single still image [60-69]. Specifically, the high correlation within the sequence of images helps remove artifacts from two perspectives. In the first place, it expands the search regions from the two-dimensional image neighborhoods to the three-dimensional spatial neighborhoods, thereby providing 
additional information which can be used to restore the reference image. Secondly, utilizing the consecutive CT images can be beneficial to remove artifacts as the residual error in each adjacent image is correlated.

Based on the analysis above, we notice the similarity between the task of artifact removal between successive images and the task of video denoising. Due to this similarity and the lack of relevant deep learning-based 3D CT reconstruction algorithms, we investigate lots of current prevailing research works in video denoising [60-69], and find out that these state-of-the-art methods give great prominence to motion estimation due to the strong redundancy along the motion trajectories. Therefore, we need a structure that can not only look into the three-dimensional spatial neighborhood, but can also conduct motion estimation between these consecutive images, so as to productively remove artifacts from limited-view CT images.

\subsection{Overall Structure}

\subsubsection{Stage One: Data Restoration in the Radon Domain}

In this stage, we propose an AAE as shown in Figure 3, which is composed of an autoencoder and a discriminator. The parametric architecture of the autoencoder can be seen from Table 1, it incorporates an encoder and a decoder that are highly symmetrical. In the encoder, each building block (refers to Figure 4) extracts representative features and is followed a Maxpool Layer that conducts downsampling. Each downsampling here will halve the height and width of the activation map and double the number of channels, and the $I C$ and $O C$ stand for the number of input channels and output channels of these building blocks and layers. After obtaining the high-level semantic features from this encoder, we establish a decoder for image texture restoration. Transposed convolution is adopted here for feature upsampling with its stride and kernel size both equal to 2 , each upsampling here will double the height and width of the activation map and halve the number of channels.

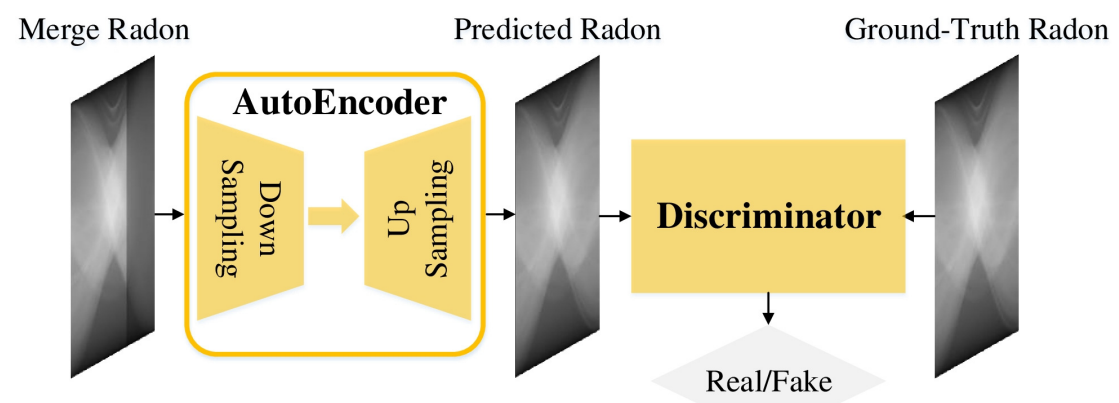

Figure 3. The overall architecture of our proposed AAE in stage one.

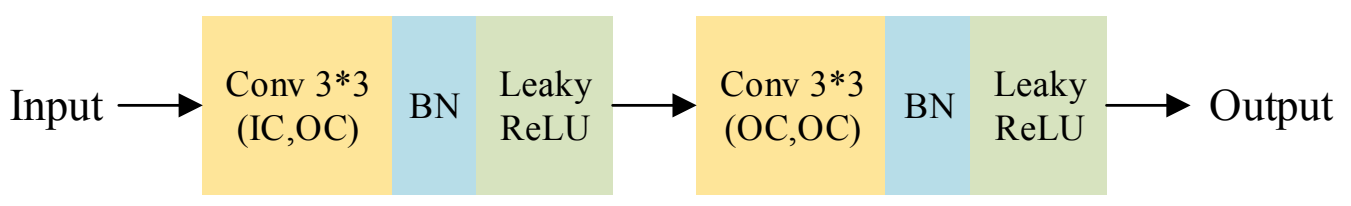

Figure 4. The diagram of the building block in AAE.

Besides, skip connections [40] are added between feature maps with the same resolution in the encoder and decoder. In the encoder, in order to acquire high-level semantic features, we conduct multiple downsampling which leads to the final feature map with a relatively low resolution, and makes it difficult for the decoder to restore the image texture. Thus, we need to utilize skip connections that can incorporate low-level features from the encoder which can help accurately precise image inpainting. It has been proved that, this sort of multi-scale, U-Net-like architectures can be well applied to medical image processing. 
Table 1. Parametric Structure of the AAE.

\begin{tabular}{|c|c|c|c|c|c|}
\hline Layer & IC & $O C$ & Stride & $\begin{array}{l}\text { Input } \\
\text { Size }\end{array}$ & $\begin{array}{l}\text { Output } \\
\text { Size }\end{array}$ \\
\hline Block1 & 1 & 32 & 1 & $192 \times 512$ & $192 \times 512$ \\
\hline Pool1 & 32 & 32 & 2 & $192 \times 512$ & $96 \times 256$ \\
\hline Block2 & 32 & 64 & 1 & $96 \times 256$ & $96 \times 256$ \\
\hline Pool2 & 64 & 64 & 2 & $96 \times 256$ & $48 \times 128$ \\
\hline Block3 & 64 & 128 & 1 & $48 \times 128$ & $48 \times 128$ \\
\hline Pool3 & 128 & 128 & 2 & $48 \times 128$ & $24 \times 64$ \\
\hline Block4 & 128 & 256 & 1 & $24 \times 64$ & $24 \times 64$ \\
\hline Pool4 & 256 & 256 & 2 & $24 \times 64$ & $12 \times 32$ \\
\hline Block5 & 256 & 512 & 1 & $12 \times 32$ & $12 \times 32$ \\
\hline Up_Conv6 & 512 & 256 & 2 & $12 \times 32$ & $24 \times 64$ \\
\hline Block6 & $\begin{array}{c}256+256 \\
\text { (Concat) }\end{array}$ & 256 & 1 & $24 \times 64$ & $24 \times 64$ \\
\hline Up_Conv7 & 256 & 128 & 2 & $24 \times 64$ & $48 \times 128$ \\
\hline Block7 & $\begin{array}{l}128+128 \\
\text { (Concat) }\end{array}$ & 128 & 1 & $48 \times 128$ & $48 \times 128$ \\
\hline Up_Conv8 & 128 & 64 & 2 & $48 \times 128$ & $96 \times 256$ \\
\hline Block8 & $\begin{array}{c}64+64 \\
\text { (Concat) }\end{array}$ & 64 & 1 & $96 \times 256$ & $96 \times 256$ \\
\hline Up_Conv9 & 64 & 32 & 2 & $96 \times 256$ & $192 \times 512$ \\
\hline Conv9_1 & $\begin{array}{c}32+32 \\
\text { (Concat) }\end{array}$ & 32 & 1 & $192 \times 512$ & $192 \times 512$ \\
\hline Conv9_2 & 32 & 12 & 1 & $192 \times 512$ & $192 \times 512$ \\
\hline Conv9_3 & 12 & 1 & 1 & $192 \times 512$ & $192 \times 512$ \\
\hline
\end{tabular}

As for the discriminator, its structure is almost the same as the encoder above, except that its Block 5 has three layers whose OCs are 512, 64 and 1 respectively. The output of Block 5 is then flattened and fed into sigmoid function for probability prediction, which we average to get the final output that represents the input image's probability to be a real image. This discriminator is added to strengthen the model's ability to restore the detailed texture of images.

\subsubsection{Stage Two: Image Restoration Based on Spatial Correlation}

After data completion in the Radon domain, we manage to mitigate the severe image artifacts to a certain extent (the specific visualized result can be seen from Figure 10 in Section 3). Nevertheless, the reconstruction result still needs to be further restored to thoroughly eliminate the artifacts and present the image texture. Therefore, we need an architecture that can effectively utilize the existing information to restore these CT images. As we mentioned above, almost all the current prevailing methods merely concentrate on a single CT images while ignoring the abundant spatial information between these consecutive CT images. Therefore, in this stage, we need to establish a model that can make full use of the spatial correlation. Recalling the discussion in II.A, we learn that this model should be capable digging into the three-dimensional spatial neighborhood and capturing motion between the continuous CT images.

Generally, an explicit motion estimation stage would have a relatively large memory cost, which may cause certain obstacles to its application. However, the two-step cascaded architecture in [70] appears to inherently embed the motion of objects with high efficiency. 
Enlightened by this, we establish the Spatial Adversarial Autoencoder that consists of the Spatial Autoencoder and the discriminator (its structure is the same as it is in stage one), the overall structure of the Spatial-AAE can be seen from Figure 5.

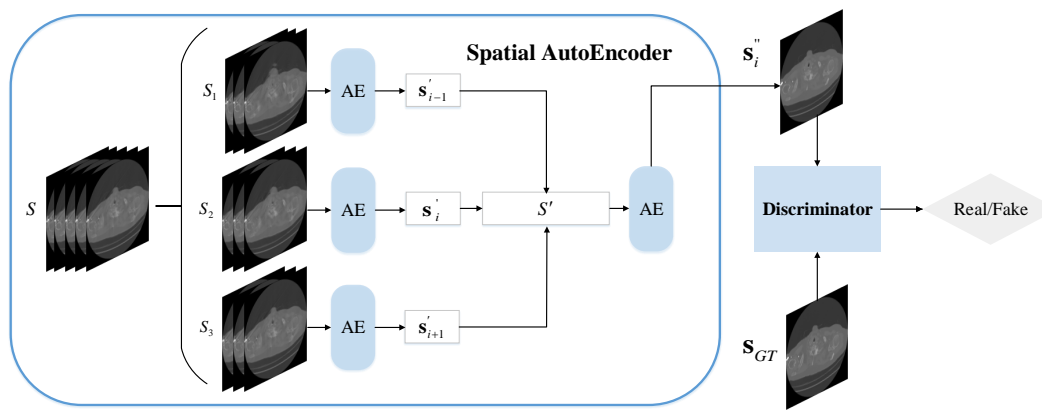

Figure 5. The overall architecture of our proposed Spatial-AAE in stage two.

The input of the Spatial Autoencoder is five consecutive CT images $S=\left\{\mathbf{s}_{i-2}, \mathbf{s}_{i-1}, \mathbf{s}_{i}\right.$, $\left.\mathbf{s}_{i+1}, \mathbf{s}_{i+2}\right\}, S$ is divided into three sets of image sequences $S_{1}=\left\{\mathbf{s}_{i-2}, \mathbf{s}_{i-1}, \mathbf{s}_{i}\right\}$, $S_{2}=\left\{\mathbf{s}_{i-1}, \mathbf{s}_{i}, \mathbf{s}_{i+1}\right\}$ and $S_{3}=\left\{\mathbf{s}_{i}, \mathbf{s}_{i+1}, \mathbf{s}_{i+2}\right\}$. Then, they are fed into the AE block respectively, and their output is concatenated as $S^{\prime}$, which is sent into the AE block (trained separately from the AE block in the first step) to obtain the final restoration result. This whole structure can be expressed as Equation (1), where F represents the Spatial Autoencoder and $G$ stands for the AE block. The specific details of the AE block can be seen from Table 1.

$$
\mathbf{s}_{i}^{\prime \prime}=F(S)=G\left(G\left(S_{1}\right), G\left(S_{2}\right), G\left(S_{3}\right)\right)
$$

\subsubsection{Stage 3: Image Refining on Patches}

After the above two stages of hybrid-domain restoration, the limited-view CT reconstruction result can reach a relatively satisfying degree (the specific visualized result can be seen from Figure 10 in Section 3). Nevertheless, the image texture is still not precise enough compared to the ground truth CT images, thereby need to be further refined. In this stage, we utilize the idea of "coarse to fine" in deep learning, and propose the Refine Adversarial Autoencoder to refine the coarse results obtained from the second stage. The overall structure of the Refine-AAE is shown in Figure 6, which is composed of the Refine Autoencoder and the discriminator (its structure is the same as it is in stage one). More importantly, we crop the input image into four patches of the same size and adjust them to the same pattern, so that it would be easier for the model to learn this mapping from this fixed pattern.

Specifically, given the input image $\mathbf{I}_{\text {input }}$, the Refine Autoencoder firstly divides it into four patches, then use horizontal and vertical flip to convert them into the same pattern. After this, the patches are concatenated into sequence $\left\{\mathbf{I}_{p 1}, \mathbf{I}_{p 2}, \mathbf{I}_{p 3}, \mathbf{I}_{p 4},\right\}$ and fed into our AE block for texture refinement. we obtain the prediction result $\left\{\mathbf{I}_{p 1}^{\prime}, \mathbf{I}_{p 2}^{\prime}, \mathbf{I}_{p 3}^{\prime}, \mathbf{I}_{p 4}^{\prime}\right\}$ and integrate it into $\mathbf{I}_{\text {pred }}$, then it is combined with the ground truth CT image $\mathbf{I}_{G T}$ into pair for discriminator's judgment.

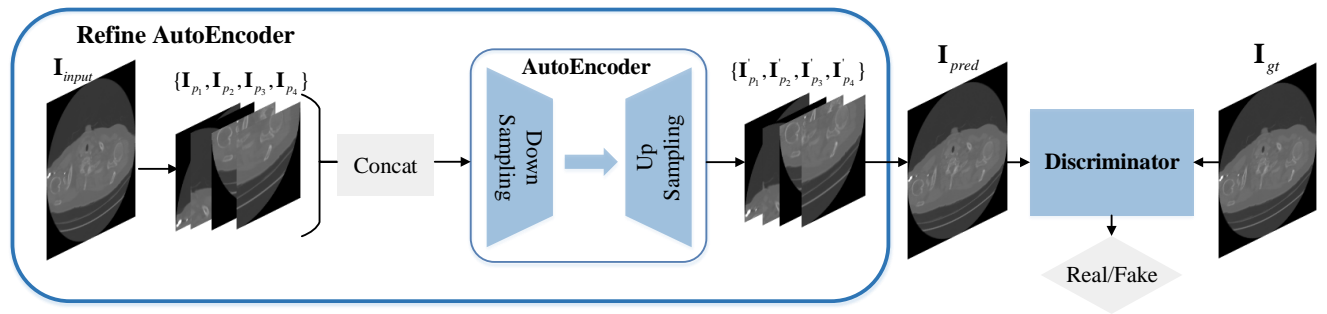

Figure 6. The overall architecture of our proposed Refine-AAE model in stage three. 


\subsection{Network Training}

All these stages are optimized separately with Adam [71] (set the learning rate to $1 \times 10^{-4}$ at the beginning), and we adopt the multi-loss function for all the autoencoders in these neural networks, the loss function is composed of $l_{M S E}, l_{A d v}$ and $l_{\text {Reg }}$ with their respective hyperparameters $\alpha_{1}, \alpha_{2}$ and $\alpha_{3}$ set to $1,1 \times 10^{-3}$, and $2 \times 10^{-8}$ respectively during training.

$$
l_{A E}=\alpha_{1} l_{M S E}+\alpha_{2} l_{A d v}+\alpha_{3} l_{\text {Reg }}
$$

In Equation (2), $l_{M S E}$ calculates the mean square error between the prediction result and its corresponding ground truth, this loss function is widely used in image inpainting because it can provide an intuitive evaluation for prediction results. The expression of $l_{M S E}$ is shown in Equation (3).

$$
l_{M S E}=\frac{1}{W \times H} \sum_{x=1}^{W} \sum_{y=1}^{H}\left(\mathbf{I}_{x, y}^{G T}-G_{A E}\left(\mathbf{I}^{\text {Input }}\right)_{x, y}\right)^{2}
$$

In Equation (3), $W$ and $H$ are the width and height of the input image respectively, $\mathbf{I}^{\text {Input }}$ and $\mathbf{I}^{G T}$ stand for the input image and its corresponding ground truth, function $G_{A E}$ represents the autoencoder.

In Equation (2), $l_{A d v}$ calculates the adversarial loss, which can be minimized to make the prediction result as close to the real data distribution as possible. Its expression is shown in Equation (4).

$$
l_{A d v}=1-D\left(G_{A E}\left(\mathbf{I}^{\text {Input }}\right)\right)
$$

where $\mathbf{I}^{\text {Input }}$ stands for the input image, function $D$ and $G_{A E}$ represent the discriminator and the autoencoder respectively.

In Equation (2), $l_{\text {Reg }}$ plays the role of a regularizer in our multi-loss function. As we know, noises are harmful to image inpainting, thereby we need a regularizer to smooth the image while preventing overfitting. Since TV Loss is widely used in image analysis, which can effectively reduce the variation between adjacent pixels, and the expression is shown in Equation (5).

$$
l_{\text {Reg }}=\frac{1}{W \times H} \sum_{x=1}^{W} \sum_{y=1}^{H}\left\|\nabla G_{A E}\left(\mathbf{I}_{x, y}^{\text {Input }}\right)\right\|
$$

where $W$ and $H$ stand for the width and height of the input image, $\|\cdot\|$ acquires the norm, $\nabla$ calculates the gradient, function $G_{A E}$ stands for the autoencoder, $\mathbf{I}^{\text {Input }}$ represents the input image.

As for the optimization of the discriminators in these stages, we minimize the loss function below to make the discriminators better distinguish between real and fake input images. The loss function $l_{D i s}$ can be seen from Equation (6).

$$
l_{D i s}=1-D\left(\mathbf{I}^{G T}\right)+D\left(G_{A E}\left(\mathbf{I}^{\text {Input }}\right)\right)
$$

where function $D$ and $G_{A E}$ represent the discriminator and the autoencoder, $\mathbf{I}^{\text {Input }}$ and $\mathbf{I}^{G T}$ are the input image and its corresponding ground truth. The discriminator outputs a scalar between 0 to 1 that stands for the probability of the input image being real. Therefore, minimizing $1-D\left(\mathbf{I}^{G T}\right)$ and $D\left(G_{A E}\left(\mathbf{I}^{\text {Input }}\right)\right)$ enables the discriminator to distinguish fake images (prediction results of the autoencoders) from all input images.

\section{Experiment}

We adopt the LIDC-IDRI [72] dataset and divide its 1018 cases (approximately 240,000 DCM files) into train set, validation set and test set according to the ratio of 1:1:3, and the amount of data is relatively large enough for us to train our models from scratch. We process these DCM files, read them into NumPy arrays , adopt normalization to ensure all data are 
scaled to the same range and create four sorts of limited-view CT data with varying degree of artifacts (the corresponding full-view CT data has 180 projection views). A geometry representative of a 2D parallel-beam CT scanner setup was used, and the sinogram was simulated by forward projecting the clinical images. The resolution of the CT image was $512 \times 512$ pixels, and each view of simulated sinogram was modeled with 512 bins on a $1 \mathrm{D}$ detector. In this section, we first conduct ablation studies to prove the rationality of our structural design, and then compare our method with other current methods under various limited-view CT data, exhibiting its remarkable performance and robustness. In addition, if not specifically mentioned, all the experiments in III.A are conducted with the limited-view CT data which lacks the post 60 projection views.

\subsection{Ablation Study}

\subsubsection{Data Preprocessing}

In our data preprocessing, to make full use of the finite prior information, we preliminarily complement the missing projection views of the original limited-view CT data (refers to Figure 2). Therefore, we conduct an experiment to see how much the additional information can help improve restoration results in the first stage. In this experiment, we feed the limited-view Radon data and the merged full-view Radon data into the AAE in stage one respectively, and then compare their restoration results with the corresponding ground truth, which can be seen in Table 2 and Figure 7. $O R$ and $M R$ stands for the original limited-view Radon data and the merged Radon data, $R O R$ and $R M R$ represents the restored $O R$ and the restored $M R$ from stage one.

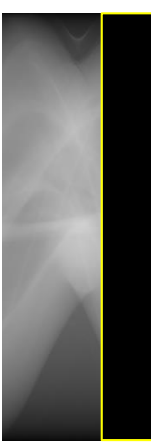

(a)

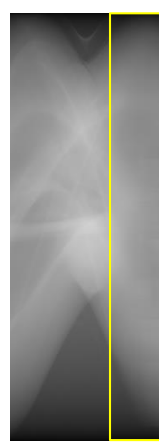

(b)

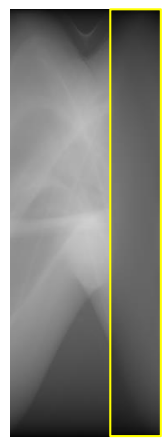

(c)

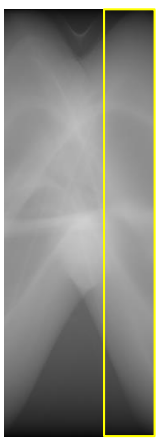

(d)

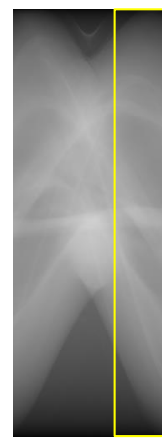

(e)

Figure 7. Visualized results obtained from different data preprocessing methods, (a) is the directly cut Radon data; (b) is the restored result of $(\mathbf{a}) ;(\mathbf{c})$ is the fused Radon data; (d) is the restored result of (c); (e) is the Radon ground truth.

Table 2. Restoration results of $\mathrm{OR}$ and $\mathrm{MR}$.

\begin{tabular}{lcccc}
\hline & OR & MR & ROR & RMR \\
\hline PSNR & 8.714 & 18.196 & 38.549 & 48.181 \\
SSIM & 0.656 & 0.936 & 0.987 & 0.995 \\
\hline
\end{tabular}

We can see from the quantitative and visualized experimental results that, $M R$ can obtain significantly better restoration outcome, and its image texture is obviously closer to the ground truth, proving the effectiveness of our data preprocessing method.

\subsubsection{The Role of Our Discriminator}

We employ our proposed discriminators in all three stages, aiming to obtain finer restoration results. Thus, we feed the merged Radon data into these two models respectively: (1) Merely the autoencoder (refers to Table 1); (2) Combination of the autoencoder and the discriminator, its quantitative and visualized experimental results can be seen from Table 3 and Figure 8. 
Table 3. Restoration results of different structures.

\begin{tabular}{ccc}
\hline & AE & AE + D \\
\hline PSNR & 40.129 & 48.181 \\
SSIM & 0.983 & 0.995 \\
\hline
\end{tabular}

We notice that the image texture of the rear 60 projection views in Figure $8 \mathrm{c}$ is obviously finer than Figure $8 \mathrm{~b}$. Also, the restoration result of structure (2) is pretty close to the ground truth as it is shown in Figure 8. Thereby, we can safely arrive at the conclusion that, the discriminator plays an important role in improving the restoration results.

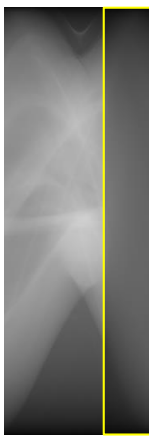

(a)

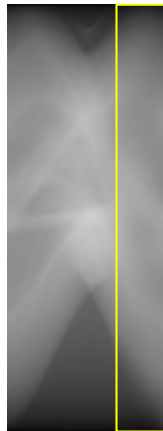

(b)

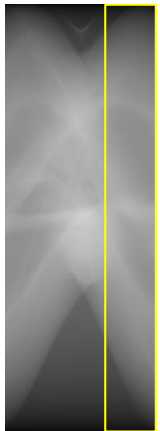

(c)

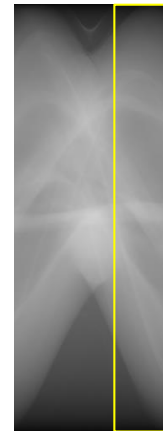

(d)

Figure 8. Visualized restoration results obtained from different data preprocessing methods, (a) is the input; (b) is the restoration result of structure (1); (c) is the restoration result of structure (2); (d) is the ground truth.

\subsubsection{The Two-Step Cascaded Architecture: Spatial-AAE}

Since the Spatial-AAE is proposed to efficaciously utilize the spatial correlation between consecutive CT images through the cascaded two-step architecture, which can manage to dig into the three-dimensional neighborhood and inherently embeds the motion of objects. To verify the effectiveness of this structural design, we carry out an experiment with reference to [70] to prove this view. In stage two, instead of feeding five successive images into Spatial-AAE, we send them directly into a single AE block (SAE) that is not capable of conduct implicit motion estimation. The experimental results can be seen from Table 4, the discriminator is also added to the SAE to ensure fairness.

Table 4. SAE vs. Spatial-AAE.

\begin{tabular}{ccc}
\hline & AAE & Spatial-AAE \\
\hline PSNR & 37.384 & 39.646 \\
SSIM & 0.929 & 0.940 \\
\hline
\end{tabular}

As we know, the AAE does not own this built-in cascade structure like Spatial-AAE to implicitly exploit the spatial correlation, it suffers from a great drop in PSNR and SSIM. This also allows us to further think about the characteristics and advantages of the Spatial-AAE architecture. Compared with AAE, Spatial-AAE adopts a two-step cascade model to implicitly perform motion estimation, and we also learned that such a process can effectively learn from residual information in consecutive images to provide additional extra prior information for restoration, thus improving the overall restoration performance to a certain extent. Besides, motion estimation needs to consume a large amount of additional computing resources in general, while such a two-step implicit motion estimation structure can manage to effectively avoids this, also create a deeper neural network to enhance the overall repair ability of the model. 
On account of these, we can safely arrive at the conclusion that, this sort of architecture can help effectively improve the restoration results.

\subsubsection{Refine the Image Texture in Patches}

In the third stage, the input image is divided and concatenated, then sent to the Refine-AAE for finer restoration. We believe that refining the image texture in patches makes it easier for the model to learn the mapping and obtain better restoration results. In addition, we want to verify the effect of different patch interception methods on the final restoration results.

To prove the above points, we design an experiment that feeds these four types of data into the model in stage three: Method 1, randomly crop four patches (size $256 \times 256$ ) from the input image (size $512 \times 512$ ); Method 2, crop the four corners out of the input image; Method 3, crop the four corners out of the input image, and then adjust them into the same pattern through different flipping method; Method 4, no cropping. Diagrams of the first three patch interception methods are shown in Figure 9, and the corresponding quantitative restoration results can be seen from Table 5 .

Table 5. Restoration results of different patch interception methods.

\begin{tabular}{lccccc}
\hline & Random Crop & Corner Crop & Corner Crop + Flip & No Cropping \\
\hline PSNR & 39.863 & 40.209 & 40.06 & 39.948 \\
\hline SSIM & 0.941 & 0.943 & 0.942 & \\
\hline & & & & & \\
\hline
\end{tabular}

Figure 9. Methods of cropping patches in stage three.

We can see that, if the patches are randomly cropped, it would to lead to a relatively poor restoration result since the pattern of input patches are complicated. However, when we adopt corner crop (with or w/o flip), its outcome exceeds method 4 due to its fixed pattern which may be easier for neural networks to learn. In addition, it is worth mentioning that method 2 has the best performance, even surpassing method 3, which particularly employs flips to adjust patches to the same pattern. It seems that the non-flip in method 2 works in the form of data augmentation, thereby improving the restoration results.

\subsubsection{Refine the Image Texture in Patches}

We previously delved into the precise design of the overall architecture, which is divided into three successive stages. Here, to demonstrate their effectiveness, quantitative and intuitive experimental results are shown in Table 6 and Figure 10.

Table 6. Restoration results of different patch interception methods.

\begin{tabular}{ccccc}
\hline & $\begin{array}{c}\text { Original } \\
\text { Input }\end{array}$ & $\begin{array}{c}\text { Stage One's } \\
\text { Output }\end{array}$ & $\begin{array}{c}\text { Stage Two's } \\
\text { Output }\end{array}$ & $\begin{array}{c}\text { Final } \\
\text { Output }\end{array}$ \\
\hline PSNR & 22.417 & 28.960 & 39.646 & 40.209 \\
\hline SSIM & 0.812 & 0.859 & 0.940 & 0.943 \\
\hline
\end{tabular}



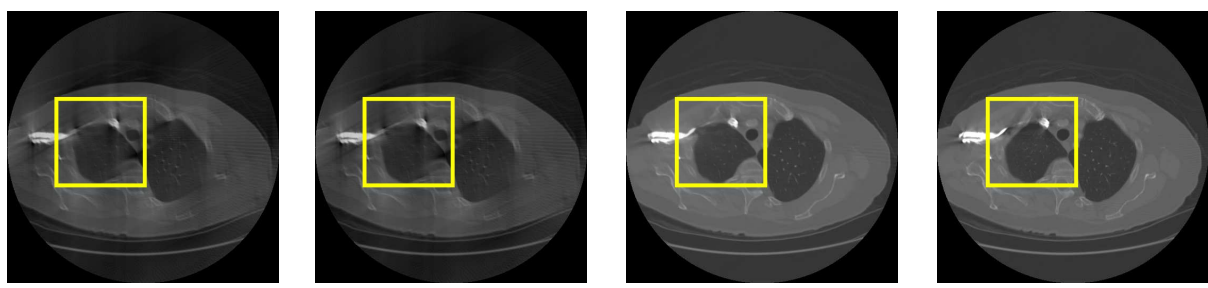

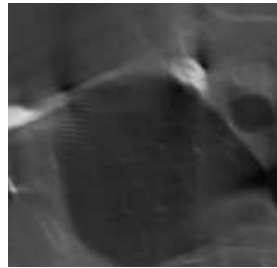

(a)

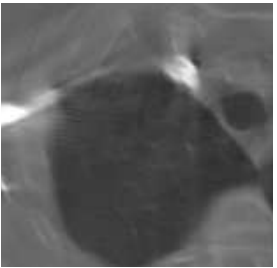

(b)

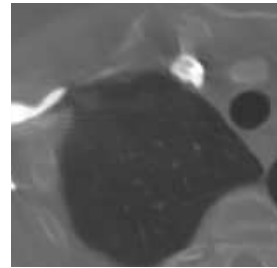

(c)

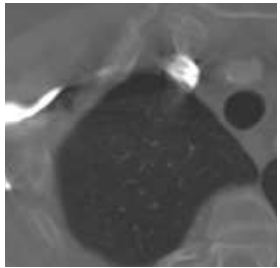

(d)

Figure 10. (a) is the original limited-view CT image, which lack the post 60 projection views (the corresponding full-view CT has 180 projection views); (b) is the CT reconstruction result of the first stage; (c) is the CT reconstruction result of the second stage; $(\mathbf{d})$ is the ground truth CT image.

As can be seen from Figure 10b, the first stage manages to alleviate the severe image artifacts, while there still remains some minor image impairments that require further improvement. Fortunately, after two stages of hybrid-domain restoration, the limited-view CT reconstruction result (refers to Figure 10c) can reach a relatively satisfying degree with no apparent artifacts. In addition, we adopt stage three to further improve the experimental results by a relatively small margin, which can also be verified by Table 6 .

\subsection{Methods Comparison}

After verifying the rationality of our structural design, and then compare our method with other current methods under various limited-view CT data. The methods include: (1) Analytical reconstruction algorithm FBP; (2) Iterative reconstruction algorithm SART combined with TV regularization; (3) Image inpainting with U-Net, after reconstructing the limited-view Radon data into images through FBP, adopt U-Net for image restoration; (4) Sinogram inpainting with U-Net, first adopt U-Net to complement the limited-view Radon data, then reconstruct it to images through FBP. In addition, in order to testify the effect of merging Radon data (MR), we implement these methods on the two sorts of input data: (1) the original limited-view Radon data; (2) the merged full-view Radon data (data preprocessing). For limited-view CT data which lack the post 60 projection views, the quantitative and intuitive restoration results of the above methods are shown in Table 7 and Figure 11. In Table 7, we evaluate all these methods' performance on the test set with their mean and standard deviation (std) to provide additional measurement for stability.

From the quantitative results above, we can see that MR manages to bring additional information for every method, thereby improving their performance by different margin. Besides, restoration using U-Net, which is known to be very effective in processing biomedical images, appears to be less useful in the Radon domain. In this case, our method combines these two domains to take advantage of their respective strengths, and finally obtain a extraordinary outcome that achieves the PSNR of 40.209 and the SSIM of 0.943, while exhibiting its stability on various limited-view data. More importantly, it not only improves the image quality by a large margin, but also realizes the precise restoration of image texture that few methods can achieve. To further demonstrate this, we calculate the corresponding error maps (refers to Figure 12), which exhibits the difference between the restoration results and the ground truth $\mathrm{CT}$ images. 


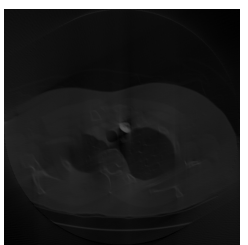

(1) FBP

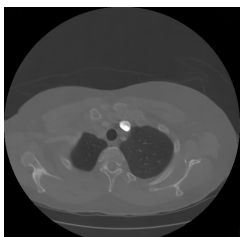

(6) II + MR

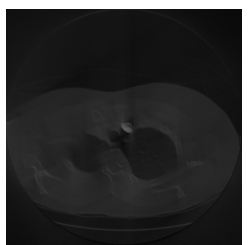

(2) FBP + MR

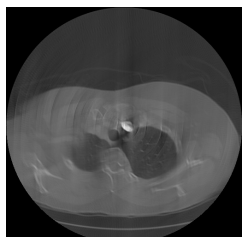

(7) SI

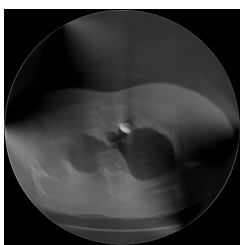

(3) SART-TV

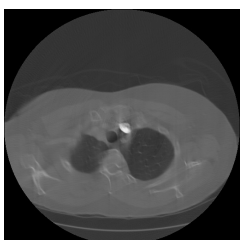

(8) SI + MR

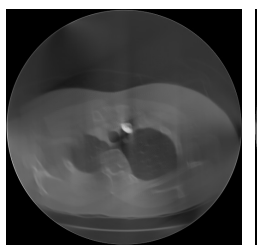

(4) SART-TV + MR

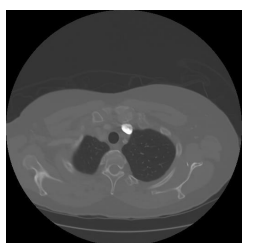

(9) Our Method

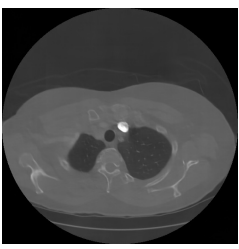

(5) II

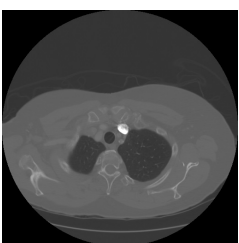

Ground Truth

Figure 11. Visualized restoration results of various methods.

Table 7. Methods Comparison.

\begin{tabular}{lcc}
\hline Algorithms & PSNR (Mean \pm Std) & SSIM (Mean \pm Std) \\
\hline (1) FBP & $11.272 \pm 0.917$ & $0.364 \pm 0.017$ \\
(2) FBP+MR & $12.354 \pm 0.811$ & $0.452 \pm 0.015$ \\
(3) SART-TV & $14.727 \pm 0.824$ & $0.635 \pm 0.021$ \\
(4) SART-TV+MR & $21.518 \pm 0.729$ & $0.807 \pm 0.019$ \\
(5) Image Inpainting (II) & $35.566 \pm 2.283$ & $0.916 \pm 0.047$ \\
(6) Image Inpainting + MR & $36.388 \pm 2.106$ & $0.927 \pm 0.047$ \\
(7) Sinogram Inpainting (SI) & $27.345 \pm 2.476$ & $0.800 \pm 0.014$ \\
(8) Sinogram Inpainting + MR & $28.960 \pm 2.461$ & $0.859 \pm 0.013$ \\
(9) Ours & $40.209 \pm 1.325$ & $0.943 \pm 0.015$ \\
\hline
\end{tabular}

Moreover, to testify the robustness of these methods, we implement them on three sorts of limited-view data that have more serious artifacts in their imaging. Including (1) limited-view CT data that lacks the middle 60 projection views; (2) limited-view CT data that lacks the middle 90 projection views; (3) limited-view CT data that lacks the middle 120 projection views, and their corresponding full-view data has 180 projection views. The experimental results can be seen from Figure 13 and Table 8.

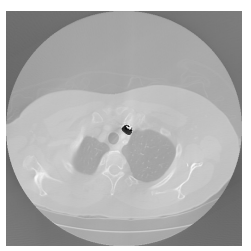

(1) FBP

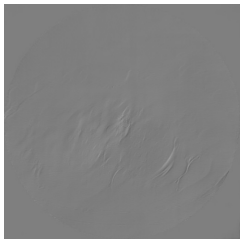

(6) II + MR

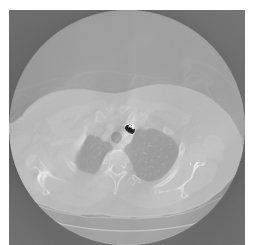

(2) $\mathrm{FBP}+\mathrm{MR}$

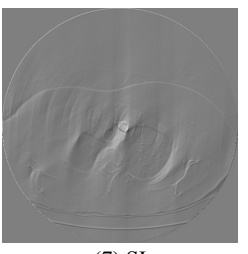

(7) SI

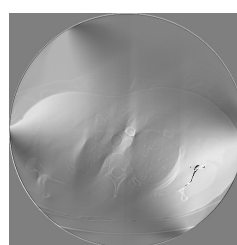

(3) SART-TV

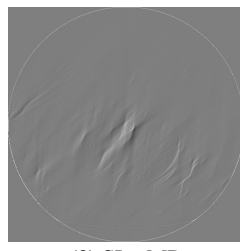

(8) SI + MR

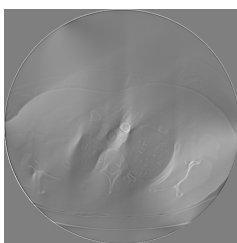

(4) SART-TV + MR

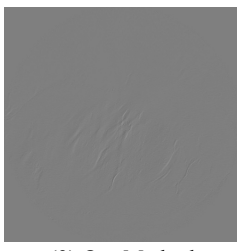

(9) Our Method

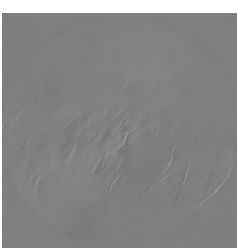

(5) II

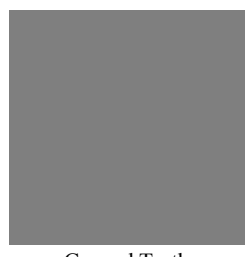

Ground Truth

Figure 12. Error maps of the restoration results obtained by various methods.

The performance of these methods has been greatly affected by the increasing information loss. Our method, however, demonstrates its outstanding robustness and still exceeds other methods by a large margin under varying degrees of damaged data. 

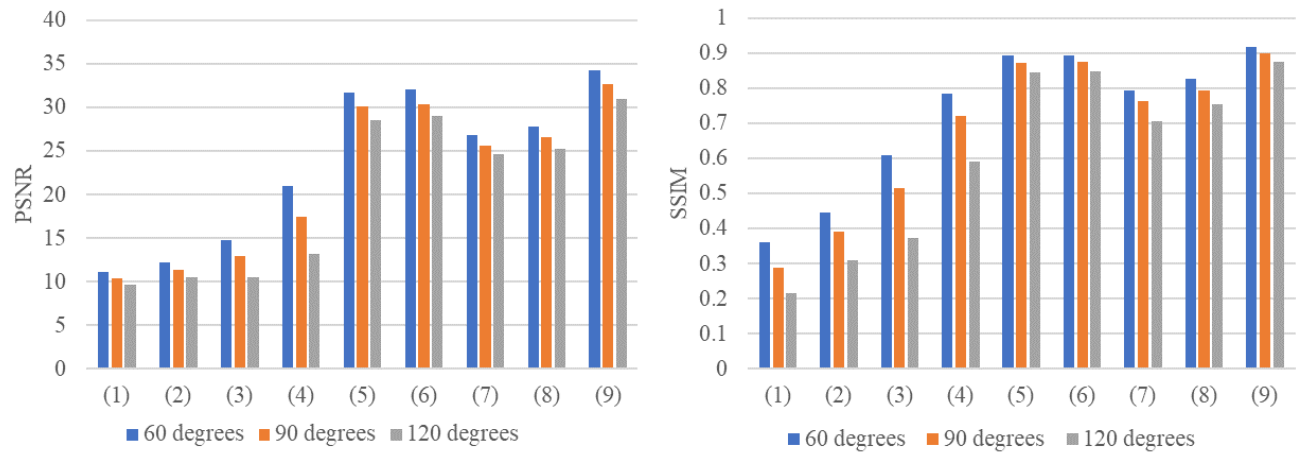

Figure 13. Histograms of different algorithms applied to different data preprocessing methods on different input data.

Table 8. Restoration results of various algorithms for limited-view data with varying degrees of artifacts.

\begin{tabular}{lcccccc}
\hline & \multicolumn{2}{c}{ CUT-MID-60 } & \multicolumn{2}{c}{ CUT-MID-90 } & \multicolumn{2}{c}{ CUT-MID-120 } \\
\hline Algorithms & PSNR & SSIM & PSNR & SSIM & PSNR & SSIM \\
\hline (1) FBP & 11.131 & 0.362 & 10.350 & 0.289 & 9.636 & 0.217 \\
(2) FBP + MR & 12.182 & 0.446 & 11.432 & 0.391 & 10.525 & 0.309 \\
(3) SART-TV & 14.758 & 0.610 & 12.945 & 0.515 & 10.492 & 0.372 \\
(4) SART-TV + MR & 21.036 & 0.784 & 17.523 & 0.722 & 13.166 & 0.592 \\
(5) Image Inpainting (II) & 31.717 & 0.895 & 30.157 & 0.873 & 28.507 & 0.846 \\
(6) Image Inpainting + MR & 32.031 & 0.895 & 30.422 & 0.876 & 28.999 & 0.849 \\
(7) Sinogram Inpainting (SI) & 26.834 & 0.793 & 25.673 & 0.763 & 24.606 & 0.705 \\
(8) Sinogram Inpainting + MR & 27.789 & 0.828 & 26.582 & 0.795 & 25.210 & 0.755 \\
(9) Ours & 34.248 & 0.919 & 32.624 & 0.900 & 30.975 & 0.876 \\
\hline
\end{tabular}

\section{Conclusions}

In order to obtain the ideal high-quality restoration results from the limited-view CT images that contains severe artifacts, we propose a hybrid-domain structure that efficaciously utilizes the spatial information between consecutive CT images, and utilizes the idea of "coarse to fine" to refine the image texture.

In the first stage, we establish an adversarial autoencoder to preliminarily complement the original limited-view Radon data. After converting the obtained full-view Radon data into images through FBP, and feed them into our proposed Spatial-AAE in stage two for artifacts removal based on spatial information. By now, we have managed to thoroughly eliminate the severe artifacts from the original limited-view CT images, while the image texture still needs to be further refined. Therefore, in the third stage, we propose the RefineAAE to refine the image in the form of patches, so as to achieve the accurate restoration of the image texture.

For limited-view Radon data that lacks the rear 60 projection views, our method can increase its PSNR to 40.209 , and SSIM to 0.943 , not only largely improve the image quality compared to other current methods, but also precisely present the image texture. At the same time, our method can be well applied to other sorts of limited-view CT data with more serious artifacts in their imaging, demonstrating its remarkable robustness.

Author Contributions: Conceptualization, K.D.; methodology, K.D.; software, K.D., C.S. and W.G.; validation, K.D., C.S. and W.G.; formal analysis, K.D. and C.S.; investigation, K.D., C.S. and W.G.; resources, K.D., C.S. and W.G.; data curation, K.D. and C.S.; writing-original draft preparation, K.D.; writing—review and editing, K.D.; visualization, W.G.; supervision, K.D., Y.L. and H.Y.; project administration, K.D., Y.L. and H.Y.; funding acquisition, Y.L. and H.Y. All authors have read and agreed to the published version of the manuscript.

Funding: This research received no external funding. 
Institutional Review Board Statement: Not applicable.

Informed Consent Statement: Not applicable.

Data Availability Statement: Not applicable.

Conflicts of Interest: The authors declare no conflict of interest.

\section{References}

1. Cormack, A.M. Representation of a Function by Its Line Integrals, with Some Radiological Applications. II. J. Appl. Phys. 1964, 35, 2908-2913. [CrossRef]

2. Hounsfield, G.N. Computerized transverse axial scanning (tomography): Part I. Description of system. Br. J. Radiol. 1973, 46, 1016-1022. [CrossRef]

3. Wang, G.; Yu, H.; Man, B.D. An outlook on x-ray CT research and development. Med. Phys. 2008, 35, 1051-1064. [CrossRef] [PubMed]

4. $\quad$ Chen, H.; Zhang, Y.; Kalra, M.K.; Lin, F.; Chen, Y.; Liao, P.; Zhou, J.; Wang, G. Low-dose CT with a residual encoder-decoder convolutional neural network. IEEE Trans. Med. Imaging 2017, 36, 2524-2535. [CrossRef] [PubMed]

5. Brenner, D.J.; Hall, E.J. Computed Tomography-An Increasing Source of Radiation Exposure. N. Engl. J. Med. 2007, 357, 2277-2284. [CrossRef] [PubMed]

6. Kalra, M.K.; Maher, M.M.; Toth, T.L.; Hamberg, L.M.; Blake, M.A.; Shepard, J.A.; Saini, S. Strategies for CT radiation dose optimization. Eur. J. Radiol. 2004, 230, 619-628. [CrossRef] [PubMed]

7. Slovis, T.L. The ALARA concept in pediatric CT: Myth or reality? Eur. J. Radiol. 2002, 223, 5-6. [CrossRef] [PubMed]

8. Krishnamoorthi, R.; Ramarajan, N.; Wang, N.E.; Newman, B.; Rubesova, E.; Mueller, C.M.; Barth, R.A. Effectiveness of a staged US and CT protocol for the diagnosis of pediatric appendicitis: Reducing radiation exposure in the age of ALARA. Radiology 2011, 259, 231-239. [CrossRef]

9. McCollough, C.H.; Primak, A.N.; Braun, N.; Kofler, J.; Yu, L.; Christner, J. Strategies for reducing radiation dose in CT. Radiol. Clin. N. Am. 2009, 47, 27-40. [CrossRef]

10. McCollough, C.H.; Bruesewitz, M.R.; Kofler, J.M. CT dose reduction and dose management tools: Overview of available options. Radiographics 2006, 26, 503-512. [CrossRef]

11. Poletti, P.A.A.; Platon, A.; Rutschmann, O.T.; Schmidlin, F.; Iselin, C.; Becker, C. Low-dose versus standard-dose CT protocol in patients with clinically suspected renal colic. Am. J. Roentgenol. 2007, 188, 927-933. [CrossRef] [PubMed]

12. Tack, D.; Maertelaer, V.D.; Gevenois, P.A. Dose reduction in multidetector CT using attenuation-based online tube current modulation. Am. J. Roentgenol. 2003, 181, 331-334. [CrossRef] [PubMed]

13. Sidky, E.Y.; Kao, C.M.; Pan, X. Accurate image reconstruction from few-views and limited-angle data in divergent-beam CT. J. X-ray Sci. Technol. 2006, 14, 119-139.

14. Chen, G.H.; Tang, J.; Leng, S. Prior image constrained compressed sensing (PICCS): A method to accurately reconstruct dynamic CT images from highly undersampled projection data sets. Med. Phys. 2008, 35, 660-663. [CrossRef] [PubMed]

15. Davison, M.E. The Ill-Conditioned Nature of the Limited Angle Tomography Problem. SIAM J. Appl. Math. 1983, 43, 428-448. [CrossRef]

16. Katsevich, A. Theoretically exact filtered backprojection-type inversion algorithm for spiral CT. SIAM J. Appl. Math. 2002, 62, 2012-2026. [CrossRef]

17. Imai, K.; Ikeda, M.; Enchi, Y.; Niimi, T. Statistical characteristics of streak artifacts on CT images: Relationship between streak artifacts and mA s values. Med. Phys. 2009, 36, 492-499. [CrossRef]

18. Liu, L. Model-based iterative reconstruction: A promising algorithm for today's computed tomography imaging. J. Med. Imaging Radiat. Sci. 2014, 45, 131-136. [CrossRef]

19. Chen, G.H.; Thériault-Lauzier, P.; Tang, J.; Nett, B.; Leng, S.; Zambelli, J.; Qi, Z.; Bevins, N.; Raval, A.; Reeder, S.; Rowley, H. Time-resolved interventional cardiac C-arm cone-beam CT: An application of the PICCS algorithm. IEEE Trans. Med. Imaging 2011, 31, 907-923. [CrossRef]

20. Rudin, L.I.; Osher, S.; Fatemi, E. Nonlinear total variation based noise removal algorithms. Physica D 1992, 60, 259-268. [CrossRef]

21. Sidky, E.Y.; Pan, X. Image reconstruction in circular cone-beam computed tomography by constrained, total-variation minimization. Phys. Med. Biol. 2008, 53, 4777. [CrossRef]

22. Niu, S.; Gao, Y.; Bian, Z.; Huang, J.; Chen, W.; Yu, G.; Liang, Z.; Ma, J. Sparse-view x-ray CT reconstruction via total generalized variation regularization. Phys. Med. Biol. 2014, 59, 2997. [CrossRef] [PubMed]

23. Andersen, A.H.; Kak, A.C. Simultaneous algebraic reconstruction technique (SART): A superior implementation of the ART algorithm. Ultrason. Imaging 1984, 6, 81-94. [CrossRef] [PubMed]

24. Xu, Q.; Yu, H.; Mou, X.; Zhang, L.; Hsieh, J.; Wang, G. Low-dose X-ray CT reconstruction via dictionary learning. IEEE Trans. Med. Imaging 2012, 31, 1682-1697. [PubMed]

25. Cao, M.; Xing, Y. Limited angle reconstruction with two dictionaries. In Proceedings of the 2013 IEEE Nuclear Science Symposium and Medical Imaging Conference (2013 NSS/MIC), Seoul, Korea, 27 October-2 November 2013; pp. 1-4. 
26. Zhang, H.; Zhang, L.; Sun, Y.; Zhang, J.; Chen, L. Low dose CT image statistical iterative reconstruction algorithms based on off-line dictionary sparse representation. Optik 2017, 131, 785-797. [CrossRef]

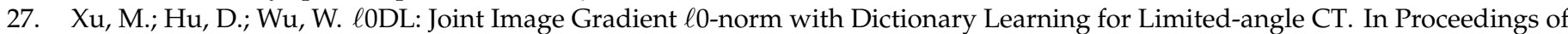
the ACM International Conference on Bioinformatics, Computational Biology and Health Informatics, Niagara Falls, NY, USA, 7-10 September 2019; p. 538.

28. LeCun, Y.; Bengio, Y.; Hinton, G. Deep learning. Nature 2015, 521, 436-444. [CrossRef]

29. He, K.; Zhang, X.; Ren, S.; Sun, J. Deep residual learning for image recognition. In Proceedings of the 2016 IEEE Conference on Computer Vision and Pattern Recognition (CVPR), Las Vegas, NV, USA, 27-30 June 2016; pp. 770-778.

30. Srivastava, R.K.; Greff, K.; Schmidhuber, J. Training very deep networks. Adv. Neural Inf. Proces. Syst. 2015, 28, $2377-2385$.

31. Xie, J.; Xu, L.; Chen, E. Image denoising and inpainting with deep neural networks. Adv. Neural Inf. Proces. Syst. 2012, 25, 341-349.

32. Dong, C.; Loy, C.C.; He, K.; Tang, X. Image super-resolution using deep convolutional networks. IEEE Trans. Pattern Anal. Mach. Intell. 2015, 38, 295-307. [CrossRef]

33. Würfl, T.; Ghesu, F.C.; Christlein, V.; Maier, A. Deep learning computed tomography. In Medical Image Computing and ComputerAssisted Intervention; Springer: Cham, Switzerland, 2016; pp. 432-440.

34. Mao, X.; Shen, C.; Yang, Y.B. Image restoration using very deep convolutional encoder-decoder networks with symmetric skip connections. Adv. Neural Inf. Proces. Syst. 2016, 29, 2802-2810.

35. Zhang, H.; Li, L.; Qiao, K.; Wang, L.; Yan, B.; Li, L.; Hu, G. Image Prediction for Limited-angle Tomography via Deep Learning with Convolutional Neural Network. arXiv 2016, arXiv:1607.08707.

36. Kang, E.; Min, J.; Ye, J.C. A deep convolutional neural network using directional wavelets for low-dose X-ray CT reconstruction Med. Phys. 2017, 44, e360-e375. [CrossRef] [PubMed]

37. Zhang, Z.; Liang, X.; Dong, X.; Xie, Y.; Cao, G. A sparse-view CT reconstruction method based on combination of DenseNet and deconvolution. IEEE Trans. Med. Imaging 2018, 37, 1407-1417. [CrossRef] [PubMed]

38. Xie, S.; Zheng, X.; Chen, Y.; Xie, L.; Liu, J.; Zhang, Y.; Yan, J.; Zhu, H.; Hu, Y. Artifact removal using improved GoogLeNet for sparse-view CT reconstruction. Sci. Rep. 2018, 8, 6700. [CrossRef]

39. Wang, J.; Liang, J.; Cheng, J.; Guo, Y.; Zeng, L. Deep learning based image reconstruction algorithm for limited-angle translational computed tomography. PLoS ONE 2020, 15, e0226963. [CrossRef]

40. Ronneberger, O.; Fischer, P.; Brox, T. U-net: Convolutional networks for biomedical image segmentation. In International Conference on Medical Image Computing and Computer-Assisted Intervention; Springer: Cham, Switzerland, 2015 ; pp. $234-241$.

41. Zhang, T.; Gao, H.; Xing, Y.; Chen, Z.; Zhang, L. DualRes-UNet: Limited Angle Artifact Reduction for Computed Tomography. In Proceedings of the 2019 IEEE Nuclear Science Symposium and Medical Imaging Conference (NSS/MIC), Manchester, UK, 26 October-2 November 2019; pp. 1-3.

42. Goodfellow, I.; Pouget-Abadie, J.; Mirza, M.; Xu, B.; Warde-Farley, D.; Ozair, S.; Courville, A.; Bengio, Y. Generative adversarial nets. Adv. Neural Inf. Proces. Syst. 2014, 27, 2672-2680.

43. Xie, S.; Xu, H.; Li, H. Artifact Removal Using GAN Network for Limited-Angle CT Reconstruction. In Proceedings of the 2019 Ninth International Conference on Image Processing Theory, Tools and Applications (IPTA), Istanbul, Turkey, 6-9 November 2019; pp. 1-4.

44. Anirudh, R.; Kim, H.; Thiagarajan, J.J.; Mohan, K.A.; Champley, K.M. Improving Limited Angle CT Reconstruction with a Robust GAN Prior. arXiv 2020, arXiv:1910.01634.

45. Li, Z.; Zhang, W.; Wang, L.; Cai, A.; Liang, N.; Yan, B.; Li, L. A sinogram inpainting method based on generative adversarial network for limited-angle computed tomography. In 15th International Meeting on Fully Three-Dimensional Image Reconstruction in Radiology and Nuclear Medicine; International Society for Optics and Photonics: Okinawa, Japan, 2019; Volume 11072, p. 1107220

46. Li, Z.; Cai, A.; Wang, L.; Zhang, W.; Yan, B. Promising Generative Adversarial Network Based Sinogram Inpainting Method for Ultra-Limited-Angle Computed Tomography Imaging. IEEE Sens. J. 2019, 19, 3941. [CrossRef]

47. Dai, X.; Bai, J.; Liu, T.; Xie, L. Limited-view cone-beam CT reconstruction based on an adversarial autoencoder network with joint loss. IEEE Access 2018, 7, 7104-7116. [CrossRef]

48. Anirudh, R.; Kim, H.; Thiagarajan, J.J.; Mohan, K.A.; Champley, K.; Bremer, T. Lose the views: Limited angle CT reconstruction via implicit sinogram completion. In Proceedings of the IEEE Conference on Computer Vision and Pattern Recognition (CVPR), Salt Lake City, UT, USA, 18-23 June 2018; pp. 6343-6352.

49. Dai, X.; Liu, T.; Hu, D.; Yan, S.; Shi, D.; Deng, H. Limited Angle Cone-Beam CT Image Reconstruction Method Based on Geometric Image Moment. CN Patent CN201510644674.X, 24 February 2016.

50. Feldkamp, L.A.; Davis, L.C.; Kress, J.W. Practical cone-beam algorithm. JOSA A 1984, 1, 612-619. [CrossRef]

51. Hammernik, K.; Würfl, T.; Pock, T.; Maier, A. A deep learning architecture for limited-angle computed tomography reconstruction. In Bildverarbeitung für die Medizin 2017; Springer: Berlin/Heidelberg, Germany, 2017; pp. 92-97.

52. Zhao, J.; Chen, Z.; Zhang, L.; Jin, X. Unsupervised Learnable Sinogram Inpainting Network (SIN) for Limited Angle CT reconstruction. arXiv 2018, arXiv:1811.03911.

53. Zhao, Z.; Sun, Y.; Cong, P. Sparse-View CT Reconstruction via Generative Adversarial Networks. In Proceedings of the 2018 IEEE Nuclear Science Symposium and Medical Imaging Conference Proceedings (NSS/MIC), Sydney, NSW, Australia, 10-17 November 2018; pp. 1-5. 
54. Lee, D.; Choi, S.; Kim, H.J. High quality imaging from sparsely sampled computed tomography data with deep learning and wavelet transform in various domains. Med. Phys. 2019, 46, 104-115. [CrossRef] [PubMed]

55. Zhang, Q.; Hu, Z.; Jiang, C.; Zheng, H.; Ge, Y.; Liang, D. Artifact removal using a hybrid-domain convolutional neural network for limited-angle computed tomography imaging. Phys. Med. Biol. 2020, 65, 155010. [CrossRef]

56. Zhou, E.; Fan, H.; Cao, Z.; Jiang, Y.; Yin, Q. Extensive Facial Landmark Localization with Coarse-to-Fine Convolutional Network Cascade. In Proceedings of the IEEE International Conference on Computer Vision (ICCV) Workshops, Sydney, Australia, 1-8 December 2013; pp. 386-391.

57. Pavlakos, G.; Zhou, X.; Derpanis, K.G.; Daniilidis, K. Coarse-to-Fine Volumetric Prediction for Single-Image 3D Human Pose In Proceedings of the 2017 IEEE Conference on Computer Vision and Pattern Recognition (CVPR), Honolulu, HI, USA, 21-26 July 2017; pp. 1263-1272.

58. Sarlin, P.E.; Cadena, C.; Siegwart, R.; Dymczyk, M. From Coarse to Fine: Robust Hierarchical Localization at Large Scale In Proceedings of the 2019 IEEE/CVF Conference on Computer Vision and Pattern Recognition (CVPR), Long Beach, CA, USA, 15-20 June 2019; pp. 12716-12725.

59. Zheng, F.; Sun, X.; Jiang, X.; Guo, X.; Yu, Z.; Huang, F. A Coarse-to-fine Pyramidal Model for Person Re-identification via Multi-Loss Dynamic Training. arXiv 2018, arXiv:abs/1810.12193.

60. Pascanu, R.; Mikolov, T.; Bengio, Y. On the difficulty of training recurrent neural networks. In Proceedings of the 30th International Conference on Machine Learning, Atlanta, GA, USA, 16-21 June 2013; pp. 1310-1318.

61. Caballero, J.; Ledig, C.; Aitken, A.; Acosta, A.; Totz, J.; Wang, Z.; Shi, W. Real-Time Video Super-Resolution with Spatio-Temporal Networks and Motion Compensation. In Proceedings of the 2017 IEEE Conference on Computer Vision and Pattern Recognition (CVPR), Honolulu, HI, USA, 21-26 July 2017; pp. 2848-2857.

62. Maggioni, M.; Boracchi, G.; Foi, A.; Egiazarian, K. Video Denoising, Deblocking, and Enhancement Through Separable 4-D Nonlocal Spatiotemporal Transforms. IEEE Trans. Image Process 2012, 21, 3952-3966. [CrossRef] [PubMed]

63. Arias, P.; Morel, J.M. Video Denoising via Empirical Bayesian Estimation of Space-Time Patches. J. Math. Imaging Vis. 2018, 60, 70-93. [CrossRef]

64. Vogels, T.; Rousselle, F.; Mcwilliams, B.; Röthlin, G.; Harvill, A.; Adler, D.; Meyer, M.; Novák, J. Denoising with kernel prediction and asymmetric loss functions. ACM Trans. Graph. 2018, 37, 124. [CrossRef]

65. Ehret, T.; Davy, A.; Morel, J.M.; Facciolo, G.; Arias, P. Model-Blind Video Denoising via Frame-To-Frame Training. In Proceedings of the 2019 IEEE/CVF Conference on Computer Vision and Pattern Recognition (CVPR), Long Beach, CA, USA, 15-20 June 2019; pp. 11369-11378.

66. Claus, M.; van Gemert, J. ViDeNN: Deep blind video denoising. In Proceedings of the 2019 IEEE/CVF Conference on Computer Vision and Pattern Recognition (CVPR), Long Beach, CA, USA, 15-20 June 2019.

67. Davy, A.; Ehret, T.; Facciolo, G.; Morel, J.M.; Arias, P. Non-Local Video Denoising by CNN. arXiv 2018, arXiv:1811.12758.

68. Tassano, M.; Delon, J.; Veit, T. DVDNET: A Fast Network for Deep Video Denoising. In Proceedings of the 2019 IEEE International Conference on Image Processing (ICIP), Taipei, Taiwan, 22-25 September 2019; pp. 1805-1809.

69. Chen, X.; Song, L.; Yang, X. Deep RNNs for video denoising. In Applications of Digital Image Processing; SPIE: Bellingham, WA, USA, 2016; Volume 9971.

70. Tassano, M.; Delon, J.; Veit, T. FastDVDnet: Towards Real-Time Deep Video Denoising without Flow Estimation. In Proceedings of the IEEE/CVF Conference on Computer Vision and Pattern Recognition (CVPR), Seattle, WA, USA, 14-19 June 2020; pp. 1354-1363.

71. Kingma, D.P.; Ba, J. Adam: A Method for Stochastic Optimization. arXiv 2017, arXiv:1412.6980.

72. Armato, S.; McLennan, G.; McNitt-Gray, M.; Meyer, C.; Reeves, A.; Bidaut, L.; Zhao, B.; Croft, B.; Clarke, L. WE-B-201B-02: The Lung Image Database Consortium (LIDC) and Image Database Resource Initiative (IDRI): A Completed Public Database of CT Scans for Lung Nodule Analysis. Med. Phys. 2010, 37, 3416-3417. [CrossRef] 